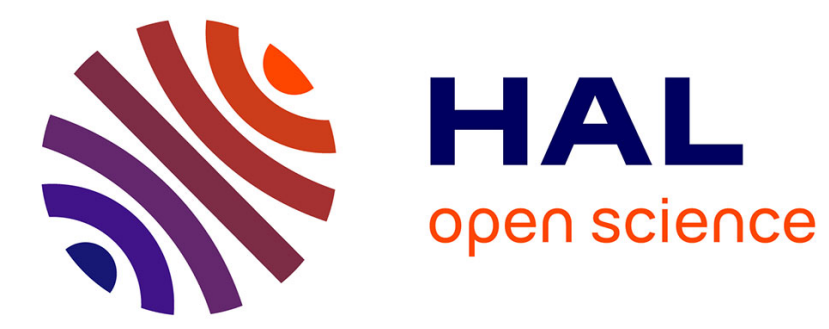

\title{
Les enceintes urbaines en moyenne Alsace (1200-1850)
}

Adrien Vuillemin

\section{To cite this version:}

Adrien Vuillemin. Les enceintes urbaines en moyenne Alsace (1200-1850). Archimède: archéologie et histoire ancienne, 2016, 3, pp.87-101. 10.47245/archimede.0003.ds1.09 . hal-01587234

\section{HAL Id: hal-01587234 \\ https://hal.science/hal-01587234}

Submitted on 13 Sep 2017

HAL is a multi-disciplinary open access archive for the deposit and dissemination of scientific research documents, whether they are published or not. The documents may come from teaching and research institutions in France or abroad, or from public or private research centers.
L'archive ouverte pluridisciplinaire HAL, est destinée au dépôt et à la diffusion de documents scientifiques de niveau recherche, publiés ou non, émanant des établissements d'enseignement et de recherche français ou étrangers, des laboratoires publics ou privés. 


\section{ARCHIMĖDE}

ARCHÉOLOGIE ET HISTOIRE ANCIENNE

\section{DOSSIER THÉMATIQUE : DES FOSSÉS ET DES REMPARTS. ENCEINTES ET SITES FORTIFIÉS DU RHIN SUPÉRIEUR ENTRE PROTOHISTOIRE ET MOYEN ÂGE}

\section{Olivier BUCHSENSCHUTZ}

Avant-propos. Des enceintes en terre anhistoriques à Google Earth

8 Lizzie SCHOLTUS

Histoire de la recherche dans le bassin de Saint-Dié-des-Vosges

20 Maxime WALTER

Les sites de hauteur du massif vosgien. Actualisation des données et modalités d'implantation

37 Jean-Jacques SCHWIEN

Chateaux et enceintes des Vosges du Nord. Topographie et longue durée

49 Anne-Marie ADAM

La palissade dans tous ses états : I'enclos du Britzgyberg (Illfurth, Haut-Rhin) et autres aménagements palissadés dans les habitats du premier âge du Fer

60 Clément FÉLIU

L'enceinte inférieure du Frankenbourg (67) et les remparts à poteaux frontaux de la fin de l'âge du Fer dans l'espace du Rhin supérieur. Pour une révision de la typologie des Pfostenschlitzmauern

74 Jacky $\mathrm{KOCH}$ et Thomas FISCHBACH

Enceintes de hauteur en pierres et formes «primitives » de châteaux ? L'exemple du Bernstein

87 Adrien VUILLEMIN Les enceintes urbaines en moyenne Alsace (1200-1850)

102 Jean-François PININGRE

Les enceintes de l'âge du Bronze et du premier âge du Fer en Franche-Comté. Un bilan des recherches

124 Clément FÉLIU et Jean-Jacques SCHWIEN

Conclusion. Nouvelles perspectives sur les enceintes du Rhin supérieur

\section{ACTUALITÉ DE LA RECHERCHE : ARCHÉOLOGIE DES RÉSEAUX}

\section{Claire CAMBERLEIN}

Les réseaux en archéologie : approche historiographique et interdisciplinaire

135 Thomas HUTIN

Lieux d'échanges et espaces publics en Gaule à La Tène finale

150 Streeve GENTNER

Économie du fer et voies de communication, de l'abattage du minerai à la distribution

du métal : I'exemple du nord de la Forêt-Noire au Ve siècle av. J.-C.

169 Loup BERNARD et Rémy WASSONG

Du Danemark au Fossé rhénan. Un siècle d'analyse des voies de communications protohistoriques : évolution des méthodes et mise en commun des données

184 Steeve GENTNER et Rémy WASSONG

Conclusion. L'archéologie des réseaux : une thématique aux multiples facettes

\section{VARIA}

187 Fábio VERGARA CERQUEIRA

To march in phalanx, to jump with weights, to tread the grapes, to knead the bread. What is the aulos for?

206 Hermann AMON

Les supra-commandements comme solution à la crise militaire du III siècle de l'Empire romain sous Philippe l'Arabe et Gallien

218 Martina BONO

Il processo di Cremuzio Cordo in Dio LVII, 24, 2-4

\section{LA CHRONIQUE D'ARCHIMÈDE}

228 Frédéric COLIN (éd.)

La Chronique d'Archimède. Bilan des activités scientifiques 2015-2016 de I'unité mixte de recherche 7044 


\title{
LES ENCEINTES URBAINES EN MOYENNE ALSACE (1200-1850)
}

\author{
Adrien VUILLEMIN \\ Docteur en histoire et archéologie, médiévale et moderne \\ Archéologue au Pôle d'Archéologie Interdépartemental Rhénan \\ UMR 7044 Archimède \\ adrien.vuillemin@pair-archeologie.fr
}

RÉSUMÉ

Cette étude porte sur les systèmes défensifs d'une quarantaine de villes petites et moyennes, édifiés en Alsace centrale entre les $\mathrm{XIII}^{\mathrm{e}}$ et $\mathrm{XV}^{\mathrm{e}}$ siècles, jusqu'à leur déclassement ou démantèlement définitif au XIX ${ }^{\mathrm{e}}$ siècle. Les ressources sollicitées sont de natures diverses: prospections des vestiges conservés, sondages archéologiques, documentation iconographique (plans, gravures, photographies anciennes), archives médiévales et modernes. Parmi ces dernières, une grande enquête sur l'état de conservation desenceintes des petites villes, bourgs et villages d'Alsace, initiée par le directeur des fortifications d'Alsace en 1779 , livre un tableau exhaustif des systèmes fortifiés avant leur abandon.

Les questions abordées portent sur les matériaux de construction, les diverses composantes de la défense (portes, murs, fossés, remparts), les données topographiques et la chronologie des aménagements. Les enseignements majeurs, dans un domaine où seules les enceintes des grandes villes ont jusqu'à présent attiré l'attention, sont la diversité des réponses apportées au besoin de défense, mais également celle des chronologies et types de structures, qui n'ont rien à envier à ces grandes villes - tout au moins jusqu'au $\mathrm{XVI}^{\mathrm{e}}$ siècle ; car sauf exception, ces villes petites et moyennes n'ont pas pu prendre le virage du bastionnement des fortifications. Elles ont en revanche assez bien entretenu les structures

Mots-CLÉs

Fortification,

ville, tour-porte, tour de flanquement, muraille,

fossé,

rempart,

barbacane, enquête militaire, défense. héritées du Moyen Âge pour encore pouvoir être considérées comme des points d'appui dans la défense de la région par l'administration royale peu avant la Révolution.
This study deals with the defensive systems of about forty small and middle-sized towns of central Alsace, from their building between the $13^{\text {th }}$ and $15^{\text {th }}$ Centuries, to their definitive dismantling during the $19^{\text {th }}$ Century. Various resources were exploited: examination of existing remains, archaeological surveys, visual sources (maps, prints, old photographs), Middle Age and Modern period archives. The latter source included a major condition report on Alsace's small cities, towns and villages, initiated by the province's head of fortifications in 1779, which offers a complete overview of fortification systems before they were abandoned.

The study addresses the questions of the construction materials used, the variety of defensive elements (doors, walls, moats, ramparts, etc.), topographical data and a chronology of their construction.

The major teachings, in a field so far focused on large cities' walls, are the broad range of solutions to the protective needs and the diversity of their chronology and layout models that are just as interesting as those of larger cities. This is noted until the $16^{\text {th }}$ Century, when, with few exceptions, these small and middle-sized towns were not able to upgrade their defense to the level of bulwarks. Well maintained though, they were still seen as a major defensive support for the region by the royal administration, soon before the French revolution.
KeYwords Urban fortifications, towns, gate tower, flanking tower city wall, ditch, rampart, barbican, military inspection, defense. 
Notre étude porte sur les systèmes défensifs d'une quarantaine de villes petites et moyennes, édifiés en Alsace centrale entre les $\mathrm{XIII}^{\mathrm{e}}$ et $\mathrm{XV}$ e siècles. Elle est originale tant dans son objet - jusqu'à présent, ce sont surtout les enceintes des grandes villes (en Alsace comme souvent ailleurs) qui ont focalisé l'attention - que dans sa démarche diachronique [1] .

Le choix de la zone géographique (fig. 1) a été motivé par sa forte concentration urbaine (41 villes sur les 75 qui maillaient le territoire de l'Alsace médiévale), par la grande diversité de sous-régions naturelles qui la composent (plaine d'Alsace incluant Ried et Kochersberg, collines sous-vosgiennes et vallées vosgiennes) et par la découverte d'un ensemble de procès-verbaux de visite d'enceintes, rédigés à la demande du Ministère de la guerre vers 1779 .

En termes chronologiques, le choix a été fait d'une étude diachronique, embrassant toute la durée d'utilisation des enceintes, de leur érection entre les $\mathrm{XIII}^{\mathrm{e}}$ et $\mathrm{XV}^{\mathrm{e}}$ siècles à leur perte définitive de fonction, celle-ci intervenant bien après la fin du Moyen Âge (fig. 2).

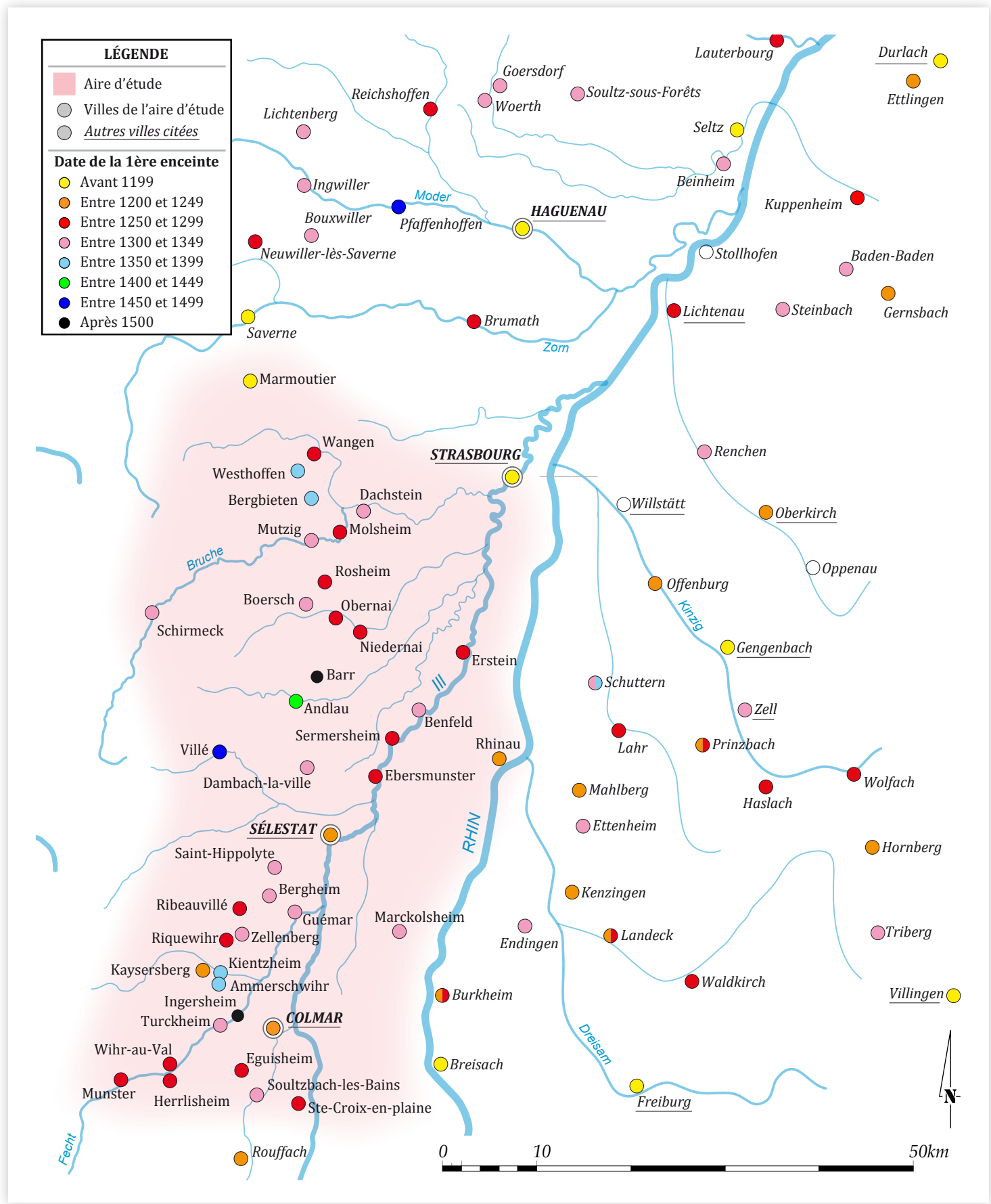

Figure 1

Carte de la zone

d'étude (d'après la carte des «Villes et marchés Rhin supérieur 12001500» du CRESAT/ Atlas historique numérique de l'Alsace - en ligne sur le site de l'Université de Haute-Alsace).

Sauf précision, toutes les illustrations de cet article sont de l'auteur.

[1] Cette recherche a donné lieu à une thèse $d$ 'histoire et d'archéologie, soutenue à I'université de Strasbourg en janvier 2015, dont nous livrons ici les principales conclusions. Voir VUILLEMIN 2015. 


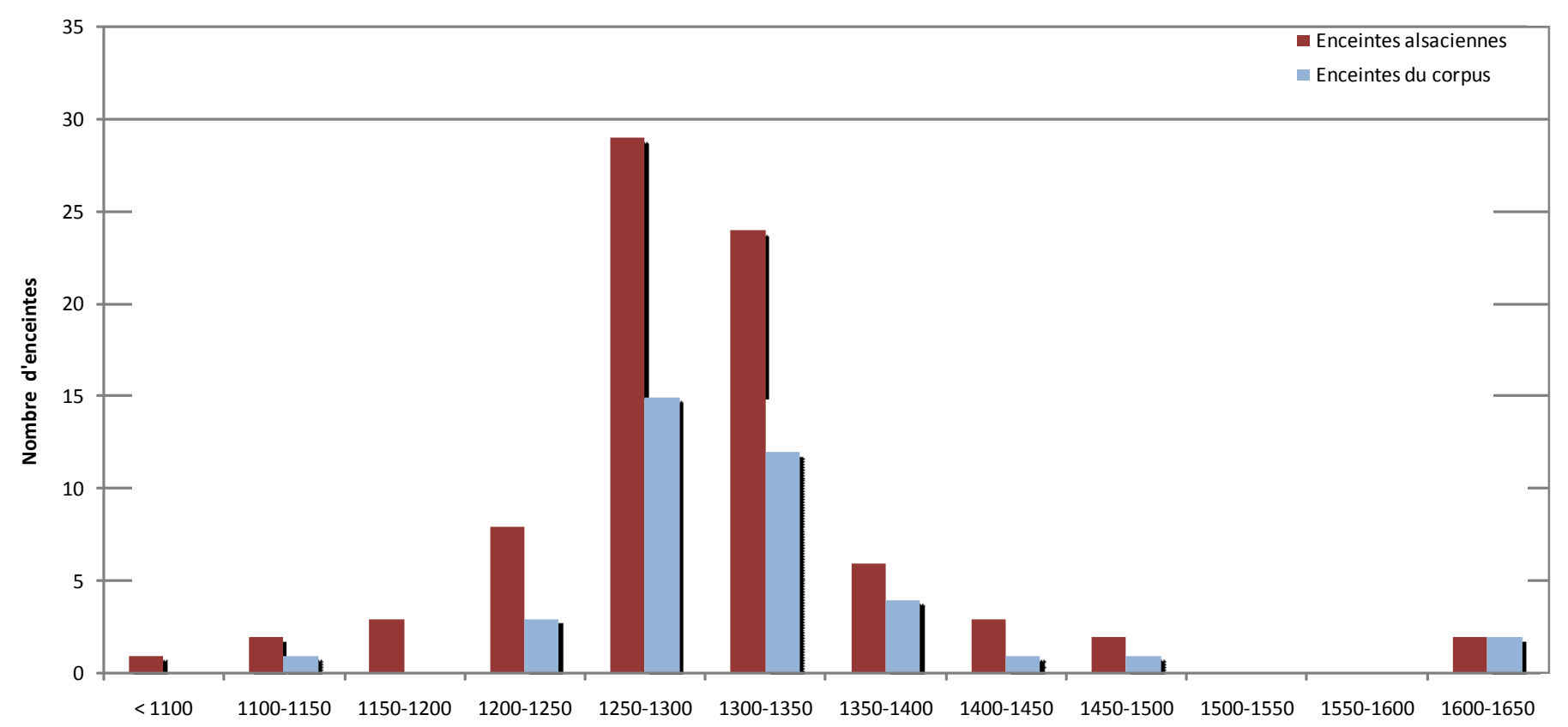

\begin{tabular}{|r|c|c|c|c|c|c|c|c|c|c|c|c|}
\hline & avant & $1100-$ & $1150-$ & $1200-$ & $1250-$ & $1300-$ & $1350-$ & $1400-$ & $1450-$ & $1500-$ & $1550-$ & $1600-$ \\
1100 & 1150 & 1200 & 1250 & 1300 & 1350 & 1400 & 1450 & 1500 & 1550 & 1600 & 1650 \\
\hline Enceintes alsaciennes & 1 & 2 & 3 & 8 & 29 & 24 & 6 & 3 & 2 & 0 & 0 & 2 \\
\hline Enceintes du corpus & 0 & 1 & 0 & 3 & 15 & 12 & 4 & 1 & 1 & 0 & 0 & 2 \\
\hline
\end{tabular}

Figure 2 : graphique de la chronologie générale de construction des enceintes, distinguant celles de notre corpus et celles du reste de l'Alsace.

Leur mise en place est sans conteste liée à un contexte politique particulier au sein du Saint-Empire romain germanique : à la fin du XII ${ }^{\mathrm{e}}$ siècle, le pouvoir impérial faiblit, entraînant la multiplication des conflits y compris dans notre région. Il s'agit de guerres privées féodales, majoritairement d'ampleur locale, n'engageant qu'un nombre d'hommes relativement limité. Elles prennent davantage la forme de pillages lucratifs des terres de l'adversaire que de sièges de châteaux ou de batailles rangées, onéreux et à l'issue incertaine. Les habitants des campagnes et leurs biens se trouvent donc être la cible privilégiée de ces conflits, ce dont ne sauraient se satisfaire ni les villageois, ni leur seigneur.

Notre terminus ante quem se situe quant à lui au XIX ${ }^{\mathrm{e}}$ siècle, au moment de la patrimonialisation des enceintes : elles perdent alors leur fonction avec, dans certains cas, leur démantèlement institutionnalisé et, dans d'autres cas, leur protection et valorisation par le service naissant des Monuments historiques. Ces bouleversements donnent lieu par ailleurs à la production d'une documentation abondante (photos, gravures, dessins, cartes et plans, etc.). Ces sources tardives apportent souvent des informations de premier plan, y compris pour le Moyen Âge, du fait de la permanence sur la longue durée de ces structures : un bel exemple en est le plan-relief de Strasbourg qui, en 1725 et avec une justesse remarquable, présente d'importants tronçons des systèmes défensifs érigés entre 1200 et 1450 puis intégrés dans les aménagements modernes.

L'ensemble s'insère bien dans la réflexion engagée par l'équipe à l'origine de cette table-ronde sur les enceintes collectives de l'espace rhénan depuis leurs origines. Certes, en comparaison de ces sites souvent protohistoriques, nos structures sont d'époque tardive mais, grâce à la qualité de la documentation, nous pouvons apporter des parties de réponse correspondant à nos questionnements transversaux sur leur rapport à une population et leurs activités, les chronologies fines, la capacité de résistance, le rôle politique et social.

\section{DE L'INTÉRÊT DES ÉTUDES DIACHRONIQUES ET DU CROISEMENT DES SOURCES}

Pour mener à bien la réflexion portant sur cette longue durée, des sources de nature très diverse ont été sollicitées : archéologiques (prospection systématique des sites du corpus, sondages de terrain, études de bâti, rapports de fouille), iconographiques et écrites (plans du Génie militaire, plans de l'administration des Ponts et Chaussées, plans cadastraux dits napoléoniens, gravures 
et dessins, photographies anciennes), médiévales (chroniques, actes juridiques, inscriptions, modernes (délibérations et règlements du Magistrat et du conseil, comptes communaux) voire contemporaines (documentation liée aux travaux d'urbanisme).

Parmi ces sources, deux ont été particulièrement riches pour notre propos.

Les procès-verbaux de visite d'enceintes et de mémoires militaires, rédigés en 1779 ou dans les années qui suivirent, sur l'ordre du secrétaire d'État à la guerre, offrent un éclairage en grande partie inédit sur l'état de conservation de fortifications médiévales à la veille de la Révolution française (fig. 3). Si plusieurs indices nous permettent d'établir que cette enquête a concerné toutes les localités alsaciennes fortifiées, les 36 procèsverbaux et 25 mémoires localisés jusqu'à présent portent en majorité sur des enceintes situées en Alsace centrale, entre la Zorn et la Fecht. Outre les descriptions précises, leur intérêt réside également dans le fait que I'administration militaire estimait que les capacités défensives de ces enceintes, pourtant « archaïques » au regard des développements techniques liés à l'évolution des armes à feu, avaient encore de l'intérêt, allant jusqu'à calculer ville par ville le nombre d'hommes de garnison nécessaires à leur défense.
Par ailleurs, les plans cadastraux dits napoléoniens rendent compte de l'organisation du parcellaire avant les grandes mutations urbanistiques intervenues à partir de la seconde moitié du XIX ${ }^{\mathrm{e}}$ siècle, avec la figuration en clair des éléments de la fortification (portes, tours, murailles) ou en négatif dans des alignements parcellaires.

Le croisement de ces différents types de sources s'avère nécessaire pour rendre compte de toutes les facettes d'un phénomène, permettant notamment de dépasser leurs lacunes respectives (fig. 4). Nous les avons transcrites sur des fiches monographiques répondant à une grille d'analyse élaborée au préalable. Chaque enceinte a aussi fait I'objet de cinq plans normalisés : un plan topographique au $1 / 35000$, le plan cadastral « napoléonien » géoajusté et vectorisé, un plan montrant l'état de conservation des différentes composantes de l'enceinte, un plan proposant une restitution de l'enceinte telle qu'elle est décrite dans les procès-verbaux et mémoires militaires de l'enquête de 1779 , et un essai de restitution topographique du développement des fortifications.

Au total, les questions abordées portent sur les matériaux de construction (la pierre, la terre, le bois ou encore le fer), les diverses composantes de la défense (les tours-portes, les tours, la muraille, les fossés, les remparts, les systèmes de défense tels qu'une avancée de porte), les données

\section{Mémoire sur les petites villes (1779) : Bergheim}

Les deux portes de la ville nommées porte d'en bas et porte d'en haut, placées au milieu des deux petits côtés de l'enceinte, sont deffendues par un réduit de forme quarrée avec un fossé sur ses trois cotés. Il y a un pont levis à chaque porte sur le fossé du reduit, et un pont en bois sur celui de l'enceinte terrassée. Le pont sur la dernière enceinte est en pierre. Les flancs de ces reduits sont très lézardés; on se propose de les reparer. ...

Pour mettre cette ville en état de faire la défense dont elle est susceptible, il faudroit : * ou faire sur le rempart une banquette elevée de 2 à 3 pieds [0,65 à $0,95 \mathrm{~m}]$ et arraser le mur de parapet à $41 / 2$ pieds $[1,45 \mathrm{~m}]$ au dessus de cette banquette, ou, si l'on craignoit de trop retrecir le rempart, baisser le parapet à $41 / 2$ pieds $[1,45 \mathrm{~m}]$ au dessus du rempart, et cependant conserver les creneaux deja faits pour decouvrir le pied du revetement, * rendre l'intérieur des tours praticables et y faire les ouvertures necessaires; * couper tous les arbres qui sont dans les fossé et au moyen desquels l'assaillant parviendroit à couvert au pied des murailles ; * approfondir le fossé du reduit de la porte d'en haut qui est encombré ;
* retablir la contrescarpe partout où elle est dégradée, et déblayer le fossé dans les endroits où il ne se trouve pas assés profond. Il en couteroit cher à la vérité pour reconstruire cette contrescarpe ecroulée, mais ce seroit aussi le meilleur moyen de defense.

600 hommes defendroient cette ville et $y$ logeroient sans trop gener les habitans.

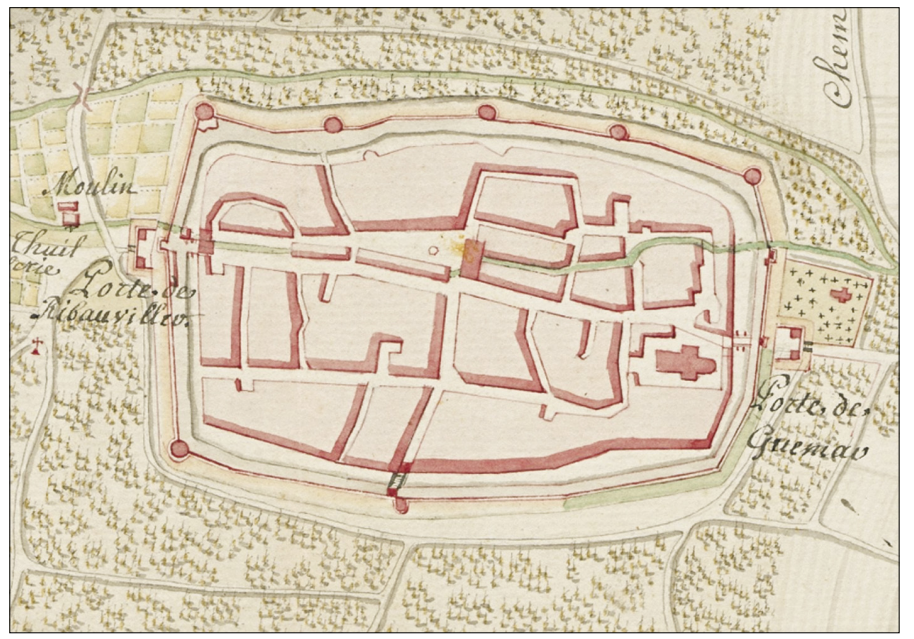

Archives municip. de Sélestat. 


\section{Mémoire sur les petites villes (1779) : Guemar}

\section{Son plan presente la figure d'un pentagone irregulier. Le developpement de son enceinte est d'environ 600 toises $[1170 \mathrm{~m}]$.}

Cette enceinte est un mur non terrassé, de 24 à 30 pieds [7,80 à 9,75 m] de hauteur exterieure, et de 15 à 18 pieds [4,90 à 5,85 m] de hauteur interieure, dans les parties qui sont encore entieres. Sa galerie est absolument detruite. Ce mur est caduc et menace ruine de toute part, il est même en deux endroits sur 50 toises [97,45 m] de long entièrement tombé, et rasé jusqu'au sol sur le côté nord-ouest qui fait face à la chaussée de Marckolsheim, en sorte qu'il n'y a d'autre defense que les maisons contigües des particuliers qui se sont trouvées derriere le mur, mais qui ont des portes et des croisées au rez de chaussée. Dans d'autres parties ce mur est si bas qu'on peut l'escalader sans echelle. A l'angle sud-ouest de la ville, le château du seigneur fait partie de l'enceinte. Il a du côté de la ville un très large fossé, et un bon revêtement. Au delà est une assés grande place nommée place du château, la seule qu'il $y$ ait dans toute la ville. On voit encore dans l'interieur de ce château un fort composé de trois tours dont les murs sont d'une prodigieuse epaisseur. Ce fort tombe en ruine, et n'est plus habité. Il ferme pourtant la ville à cet angle. Il paroit que Guemar a été autrefois un poste très fort, il y avoit en avant du fossé, qui a environ 12 à 15 toises [de 23,40 à 29,25 $\mathrm{m}$ ] de large, une enceinte de terre, en maçonnerie de chemin couvert, mais si elevée qu'elle couvroit absolument la ville. Dans cet état non seulement Guemar seroit à l'abry d'un coup de main, mais même on devroit attendre pour se rendre que l'ennemi après avoir ruiné à coup de canon le parapet du chemin couvert, qu'il ne faudroit pas manquer de palissader, et le mur

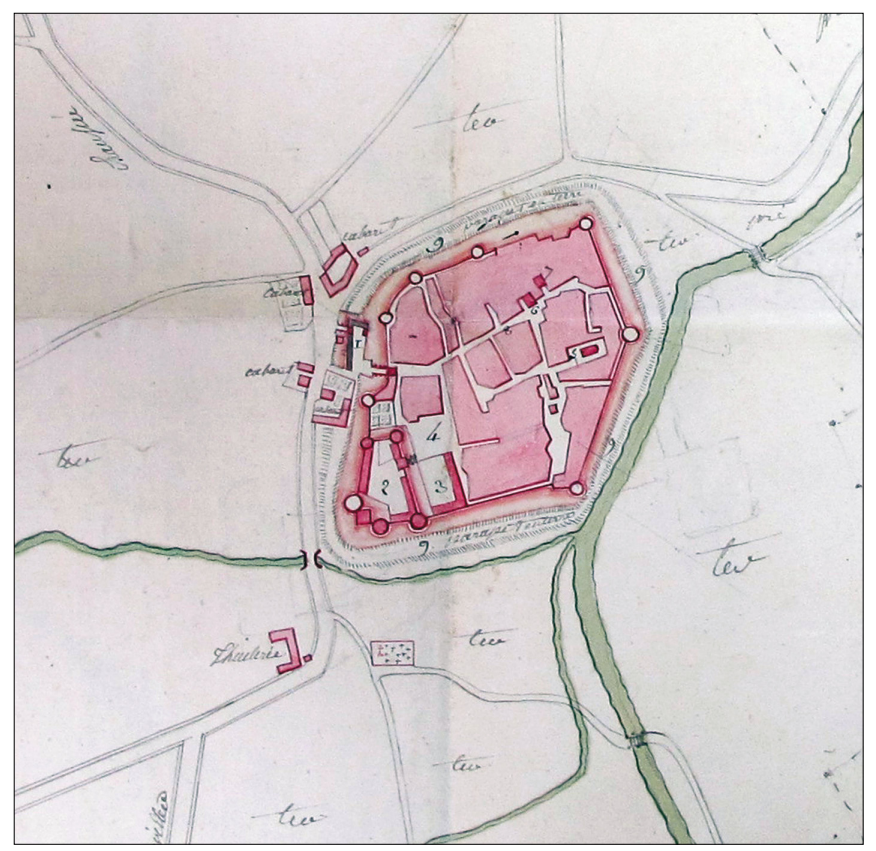

Archives municip. de Sélestat.

d'enceinte, eut traversé l'avant fossé, et se fut logé sur ce chemin couvert. On sent qu'alors l'assiegé ne sauroit s'opposer à la reconstruction des ponts, et ne devroit point tenter de defendre les brêches qu'on voit multipliées. Si l'ennemi n'avoit pas fait brêche au château, l'assiegé pourroit encore avoir la ressource de s'y retirer, en coupant le pont. Il y a un large fossé et un bon mur de revêtement. On auroit bien de la peine à le prendre sans canon, et il serviroit au moins à capituler.

...

Mais toute cette defense n'exigeroit pas moins de 500 hommes et la ville ne sauroit en loger plus de 200. topographiques (le rapport entre les surfaces encloses et les surfaces occupées par les structures défensives (fig. 5), la gestion des abords intérieurs et extérieurs de l'enceinte, la relation de l'enceinte urbaine au château, au réseau hydrographique, à la voirie et au relief) et la chronologie des aménagements.

\section{DES ENCEINTES «TYPE »}

L'un des enjeux de l'étude était de déterminer les réponses apportées par les petites et moyennes localités à leurs besoins en matière de défense. Loin d'obtenir une réponse unique, il est apparu que les structures défensives mises en œuvre avant 1600 étaient relativement diversifiées et n'avaient rien à envier aux grandes villes, que ce soit en matière de chronologie, ou de technologie. Si 16 enceintes urbaines de moyenne Alsace n'ont pas connu d'évolution notable de leur plan entre leur mise en place (première moitié du XIII ${ }^{\mathrm{e}}$ siècle - seconde moitié du XVe siècle) et la guerre de Trente Ans [2], 21 ont été étendues et/ou renforcées durant le même laps de temps. Le plan des enceintes évolue en vertu de deux phénomènes distincts : I'agrandissement et le renforcement, qui traduisent respectivement un développement urbain et une évolution technologique (fig. 6). Le second est nettement plus fréquent que le premier.

Au vu des données accumulées sur les différentes enceintes du corpus, il est possible de proposer un profil type,

[2] Il s'agit de Bergbieten, Boersch, Dambach-la-Ville, Marmoutier, Mutzig, Saint-Hippolyte, Soultzbach-les-Bains, Turckheim, Villé, Wangen, Westhoffen, Wihr-au-Val, Zellenberg. Dans les cas d'Erstein, Schirmeck, Sermersheim et Rhinau, l'état final des enceintes avant leur destruction n'est pas déterminé. 
Figure 4

Exemple de plan de synthèse de tous types de données recueillies, calées sur le fond de plan cadastral : Ingwiller.

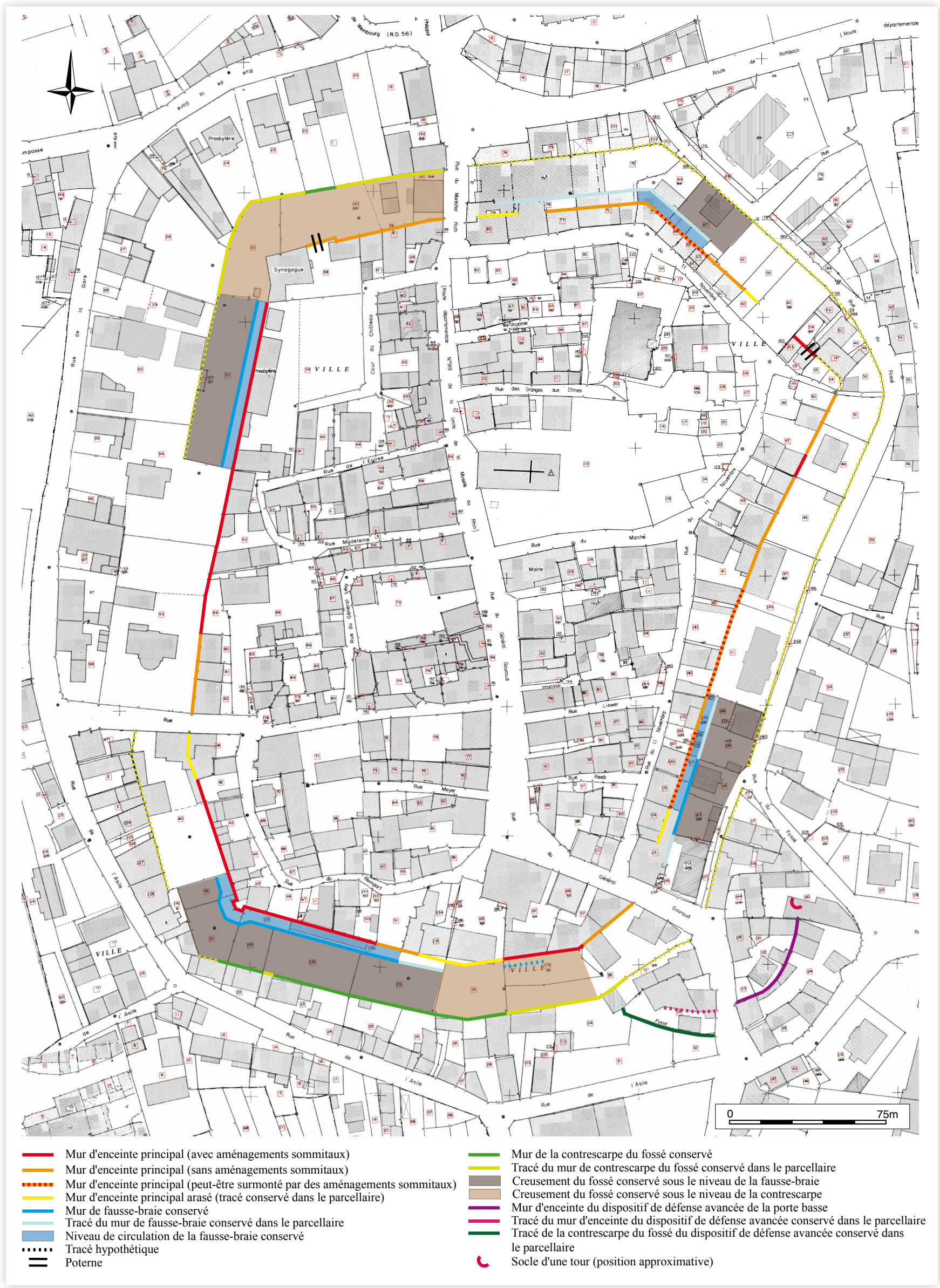




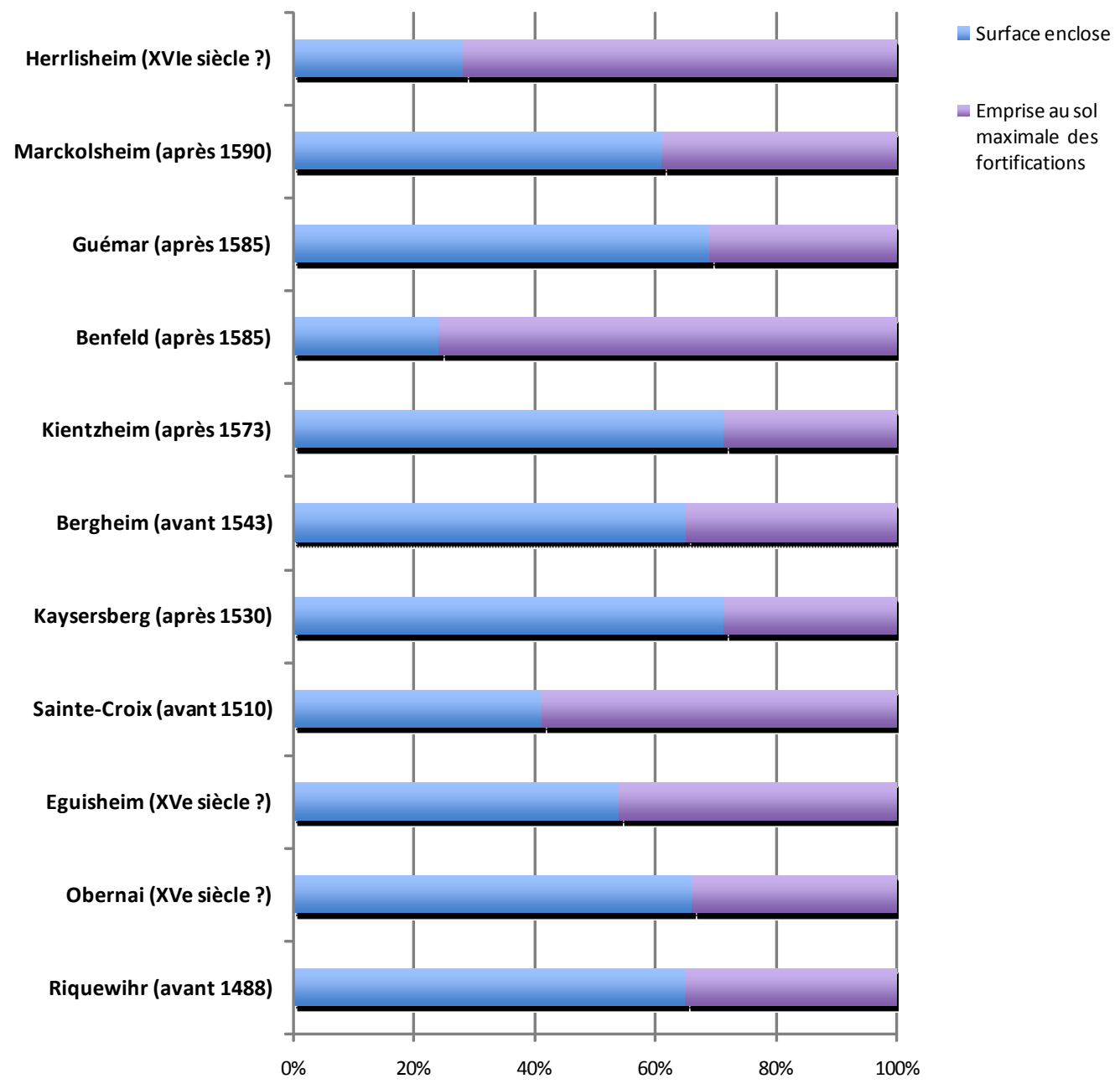

Figure 5

Diagramme du rapport entre les surfaces encloses et les extensions maximales des fortifications, avec en regard l'exemple de Herrlisheim, ce petit bourg dont l'emprise totale des fortifications est la plus importante du corpus.

\begin{tabular}{|c|c|c|c|}
\hline $\begin{array}{c}\text { Datation du } \\
\text { renforcement }\end{array}$ & VILLE & $\begin{array}{c}\text { Surface } \\
\text { enclose }\end{array}$ & $\begin{array}{c}\text { Emprise } \\
\text { au sol } \\
\text { maximale des } \\
\text { fortifications }\end{array}$ \\
\hline 1488 av. & Riquewihr & 6,06 & 3,24 \\
\hline 1499 av. & Obernai & 12,43 & 6,36 \\
\hline 1499 av. & Eguisheim & 3,43 & 2,89 \\
\hline 1510 av. & Sainte-Croix & 7,31 & 10,38 \\
\hline 1530 ap. & Kaysersberg & 11,95 & 4,79 \\
\hline 1544 av. & Bergheim & 12,56 & 6,68 \\
\hline 1573 av. & Kientzheim & 12,44 & 5,00 \\
\hline 1585 ap. & Benfeld & 6,86 & 21,20 \\
\hline 1585 ap. & Guémar & 7,38 & 3,32 \\
\hline 1590 ap. & Marckolsheim & 14,19 & 9,00 \\
\hline 1599 & Herrlisheim & 3,67 & 9,31 \\
\hline
\end{tabular}

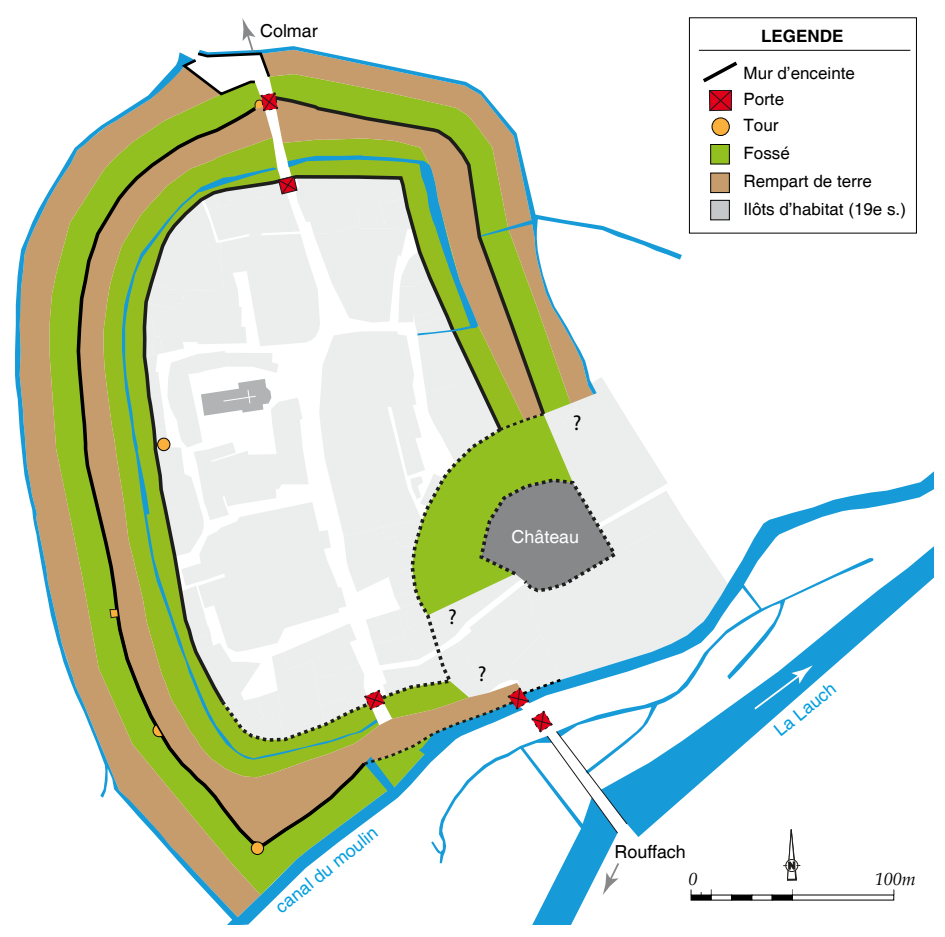




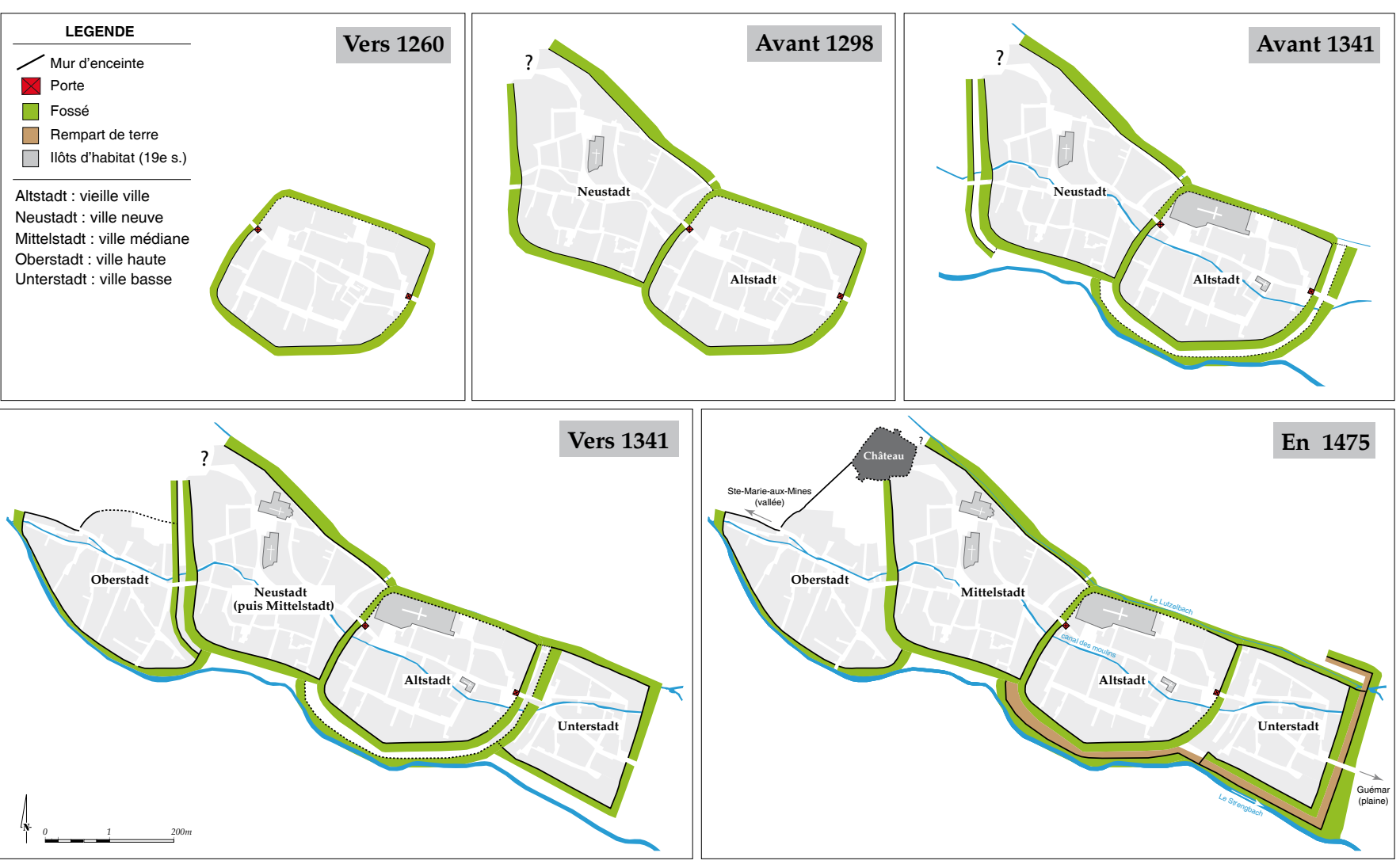

sinon idéal, et dans une moindre mesure un plan type, selon trois programmes différents : la construction entre 1300 et 1400 , une première forme de modernisation en 1500 et une seconde vers 1575 .

\section{PROFIL TYPE DES ENCEINTES CONSTRUITES ENTRE 1300 ET 1400}

L'enceinte construite entre 1300 et 1400 n'adopte pas un plan privilégié : son objectif est de ceindre la plus grande partie du bâti préexistant en faisant fi des contraintes topographiques et de leurs conséquences négatives en matière défensive. Aussi, peu importe que la localité se développe au pied d'un coteau ou soit assise à mi-pente.

Elles se développent en moyenne sur $1250 \mathrm{~m}$, pour clore un espace de 10 hectares environ.

L'érection de la muraille fait suite à l'excavation du fossé. D'une épaisseur constante de 1,10 à $1,50 \mathrm{~m}$, elle est formée d'une fourrure entre deux parements, assise sur une semelle légèrement plus épaisse. Le type de pierre utilisé en priorité dans la muraille est celui disponible aux environs immédiats de la localité. Cependant, pour les éléments architecturaux les plus remarquables, les chaînages d'angle et les arcs du passage des tours-portes par exemple, du grès fin des Vosges est utilisé. Haute depuis le fond du fossé de 5 à $7 \mathrm{~m}$, la muraille est coiffée d'un chemin de ronde composé de dalles en grès en légère saillie par rapport au parement interne de la muraille (sa largeur se situe aux alentours de 0,90 m), couvert par un mur de parapet crénelé, haut de 1,90 $\mathrm{m}$ et épais de 0,50 $\mathrm{m}$. Ce chemin de ronde est continu sur tout le pourtour de l'enceinte ; à cet effet, les murs latéraux des tours-portes et des tours de flanquement sont percés de portes; dans le cas des

\section{Figure 7}

Graphique de synthèse du plan des tours d'angle.

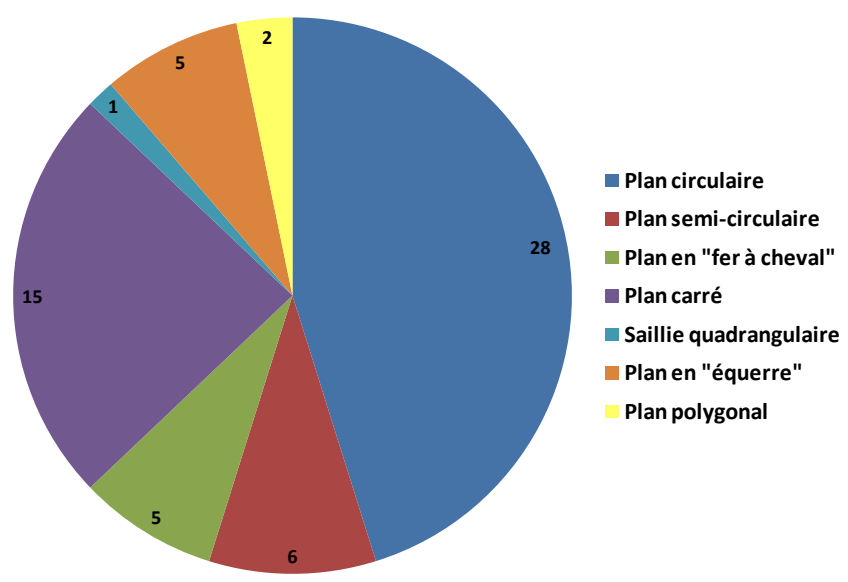


tours qui saillent uniquement sur l'extérieur, le passage est possible à l'arrière de l'édifice. L'accès au chemin de ronde s'effectue au niveau des tours-portes ou en d'autres points du pourtour de l'enceinte, par des escaliers en maçonnerie ou par le biais d'échelles.

La muraille n'est que faiblement flanquée. En prenant en compte les tours-portes, une tour est installée tous les $250 \mathrm{~m}$. Le plan circulaire (ou assimilé) est privilégié pour celles-ci (fig. 7). Leur moitié supérieure est percée d'archères.

Le fossé à fond plat se déploie immédiatement au pied de la muraille qui lui sert par conséquent d'escarpe. Il présente une largeur moyenne de $15 \mathrm{~m}$ à l'ouverture et une profondeur de 2 à $3 \mathrm{~m}$. Sa contrescarpe peut être revêtue en maçonnerie ou laissée nue. Dans le premier cas de figure, elle est quasiment verticale, présentant seulement un léger fruit de manière à supporter la poussée des terres, et dans le second cas, elle est talutée.

Pour pénétrer en ville, il faut emprunter le passage aménagé à la base de l'une des deux (ou trois) tours-portes, de plan quadrangulaire massé, réparties en des points opposés de l'enceinte. La face externe des tours-portes est soit alignée avec les courtines adjacentes, soit légèrement saillante. Le passage peut être fermé en abaissant une herse coulissant dans les rainures aménagées dans des contreforts extérieurs encadrant l'arc. La herse est complétée par des doubles vantaux. Le passage est surmonté par deux étages au moins, ouverts (sans mur) côté ville. Les murs latéraux et le mur tourné vers l'extérieur de l'étage sommital sont percés de créneaux.

Pour passer le fossé, il faut emprunter un pont formé de tabliers en bois reposant sur des piles en maçonnerie.

\section{LE PROGRAMME TYPE DES TRAVAUX DE MODERNISATION, VERS 1500}

Un programme des travaux de modernisation (et de renforcement) caractéristiques de la fin du $X V^{E}$ siècle et du début $\mathrm{du} X \mathrm{XI}^{\mathrm{E}}$ siècle peut également être proposé, selon deux formules : la première consiste uniquement à modifier les composantes de l'enceinte existante. La seconde formule, outre les modifications précitées, comprend I'adjonction d'une nouvelle ligne de défense (fig. 8).

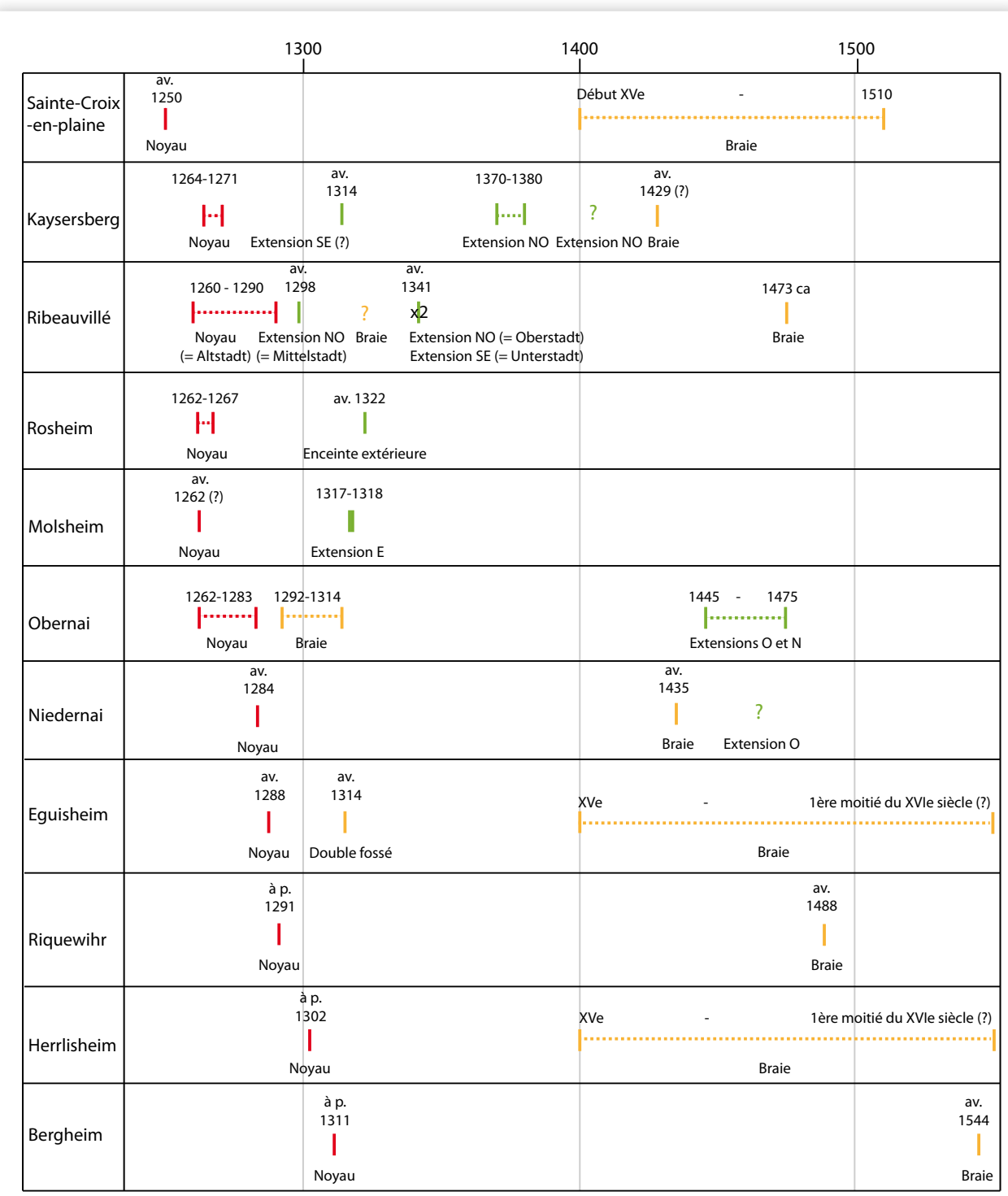

Figure 8

Exemples des formes et chronologie des réaménagements des systèmes défensifs.

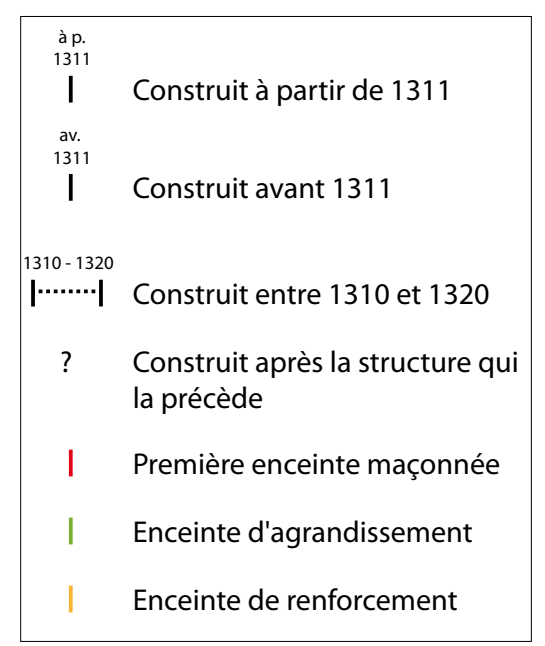


La transformation des composantes du système défensif existant vise à permettre un usage aisé des armes à feu tout en limitant I'exposition du tireur. Les créneaux rythmant le mur de parapet surmontant la muraille sont convertis en archères-canonnières et/ou en canonnières, et leur embrasure est dotée d'une barre d'appui. La mise en place d'ouvertures de tir adaptées à l'usage des armes à feu vaut aussi pour les tours, à leur niveau inférieur notamment. L'accès au passage des tours-portes est complexifié par l'installation de ponts-levis. Ils sont détachés des tours-portes, pouvant être intégrés à la structure d'avant-portes accolées.

Au-delà du fossé initial est élevé un (second) rempart. Si la topographie le permet, il enserre intégralement la première enceinte, mais quand la déclivité du terrain rend difficile, voire inutile la mise en place d'un rempart, le dédoublement de l'enceinte n'est que partiel.

Ce rempart est constitué de deux murs enserrant un terre-plein et précédé d'un nouveau fossé, à fond plat, large de $15 \mathrm{~m}$. À l'arrière, la maçonnerie est construite à l'aplomb du mur de contrescarpe du fossé initial, qui peut être revêtu à cette occasion. Le front extérieur est, lui, habillé par un mur épais de 0,60 m en moyenne, formé d'un seul parement (extérieur) et d'une fourrure. Il s'élève de 2,50 m au-dessus du niveau du terre-plein, large de 12 m environ, dont il forme le parapet. Des ouvertures de tir de type canonnière le percent régulièrement, à hauteur d'homme. Le terre-plein domine de $3 \mathrm{~m}$ le fond du fossé intérieur, et de 3,90 $\mathrm{m}$ le fond du fossé extérieur, qui est donc plus profond que le précédent. Le rempart revêtu est flanqué de tours de plan circulaire ou en fer-à-cheval, installées en priorité dans les angles formés par l'enceinte. Elles sont adaptées à l'usage de l'artillerie.

Les passages s'effectuent par des tours-portes de plan quadrangulaire massé, installées dans l'axe des tours-portes initiales. De hauteur moindre que celles-ci, leurs étages sont également ouverts du côté de la ville. Des ponts-levis à flèches y sont intégrés qui, une fois leur tablier relevé, font office de fermeture, associés ou non à deux vantaux de porte. L'accès au (nouveau) rempart depuis l'intérieur de la ville s'effectue depuis les tours-portes ou par des poternes percées (à cet effet ?) dans la muraille initiale.

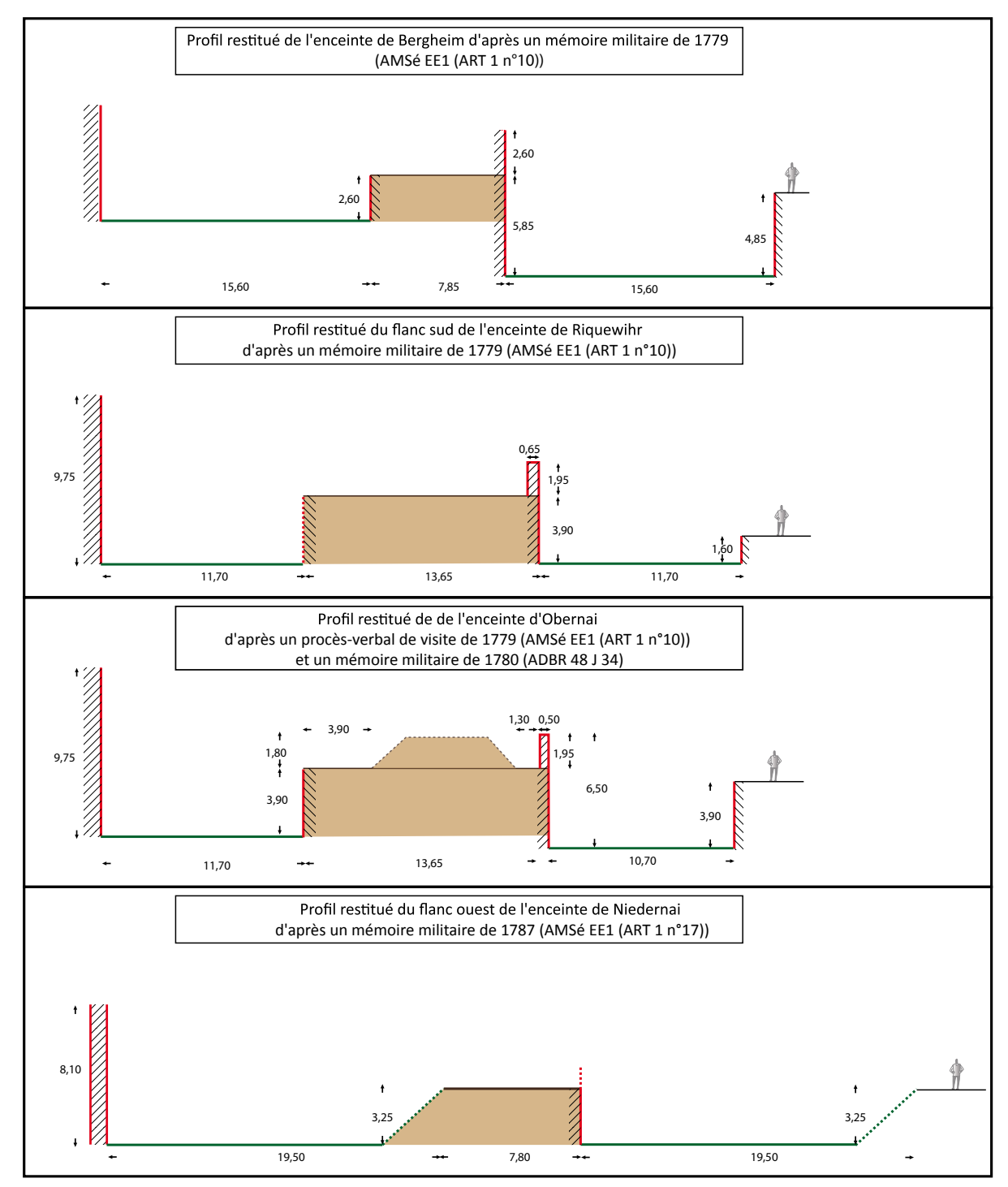

Figure 9

Exemples de profils de remparts revêtus. 


\section{LE PROGRAMME TYPE DES TRAVAUX DE RENFORCEMENT, ENTRE 1550 ET 1590}

La poursuite du programme de modernisation (et de renforcement) du système défensif se fera selon des modalités différentes à partir de 1550, privilégiant les ouvrages de terre (fig. 9).

Tout ou partie de la seconde enceinte peut voir l'adjonction d'un rempart nu qui peut être élevé jusqu'à 6,50 m (ou plus ?) au-dessus du fond des fossés qui se trouvent de part et $d$ 'autre. Une palissade et/ou une banquette de terre le coiffent. Les points d'entrée sont renforcés par la construction de barbacanes de forme grossièrement quadrangulaire, construites au-delà du (second) fossé (fig. 10). Leur porte est plus ou moins fortement désaxée par rapport au passage des tours-portes auxquelles elles donnent accès (pour éviter les tirs en enfilade).

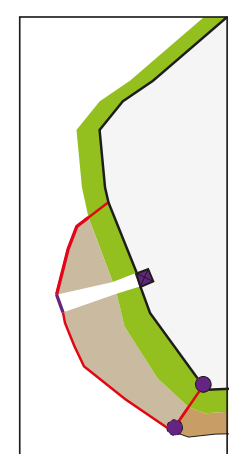

Ammerschwihr, porte haute
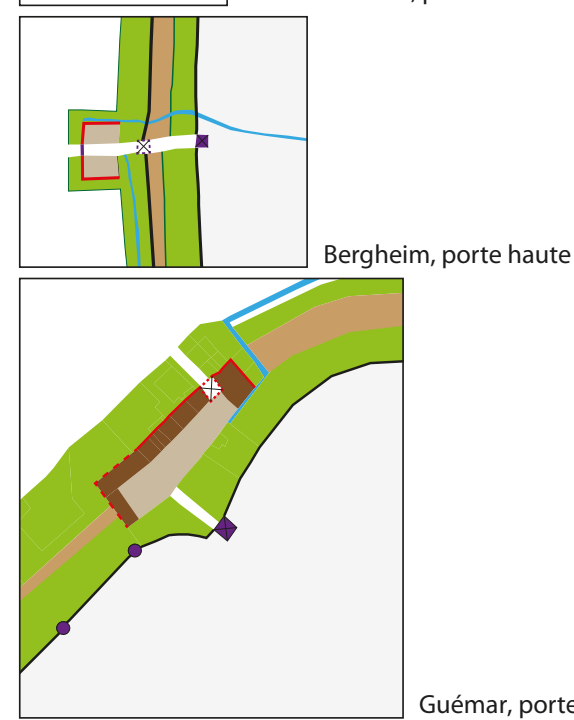

Guémar, porte haute

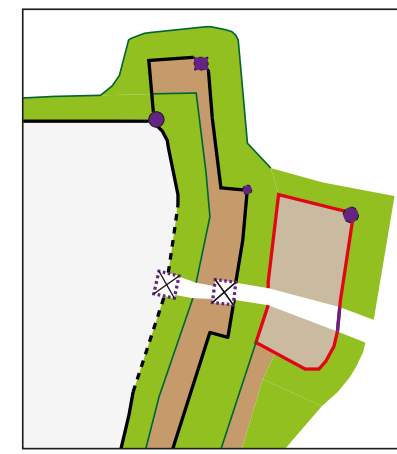

Kaysersberg, porte basse

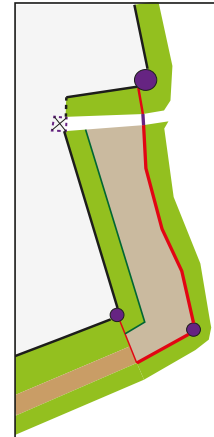

Devant la porte basse

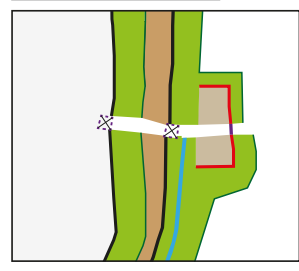

Bergheim, porte basse

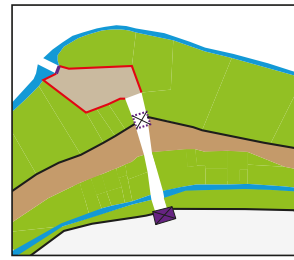

Herrlisheim, porte haute

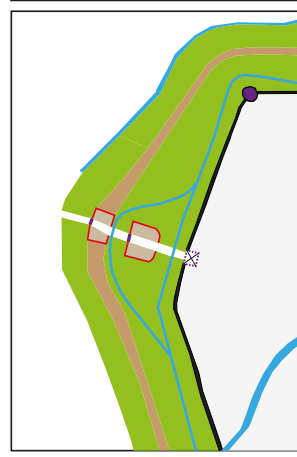

Figure 10

Exemples de barbacanes.

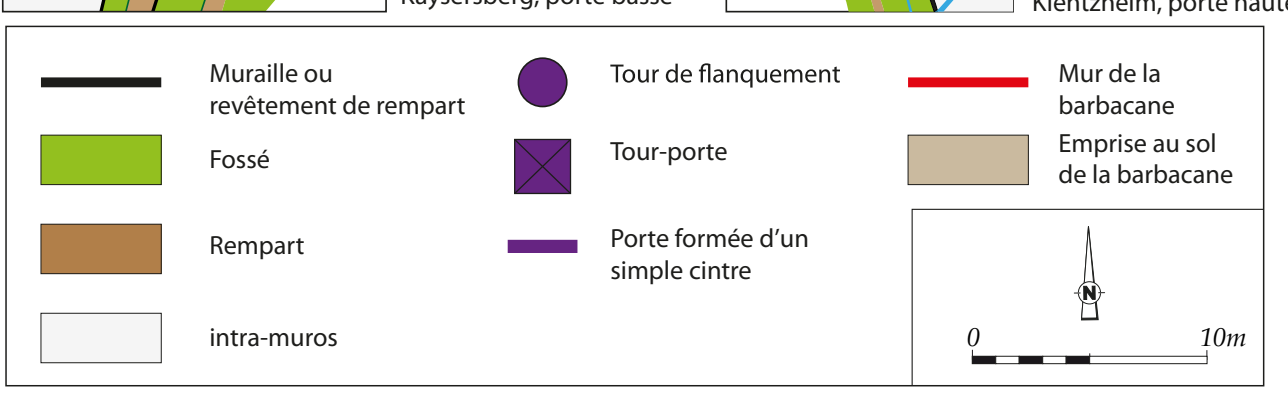


Ces trois programmes «type », fondés sur une approche statistique des différentes enceintes du corpus, donnent l'image globale d'un glissement de la pierre vers la terre, en réponse au développement de l'artillerie à poudre, en trois temps bien distincts. Cette image, schématique, demande à être nuancée : la terre a en effet pu précéder la pierre (enceintes villageoises), des braies ont d'abord pu prendre la forme de «simples » murailles, remparées dans un second temps (Obernai ?), tandis que d'autres ont pu prendre la forme de levées de terre, revêtues en maçonnerie dans un second temps (Eguisheim et Niedernai ?).

Il est d'ailleurs intéressant de constater la proximité morphologique entre les levées de terre de la fin du $\mathrm{XVI}^{\mathrm{E}}$ siècle et celles qui entrent dans la composition des enceintes villageoises, ayant pu précéder une muraille. Si elles ne répondent pas au même objectif, leur dénominateur commun pourrait être le coût réduit et le caractère aisé de la mise en œuvre.

\section{DES QUESTIONS D'ORDRE TECHNIQUE EN SUSPENS}

Qu'il s'agisse de la forme des ponts-levis, du fonctionnement des systèmes de relevage des herses, de la réalité de la mise en eau des fossés, du passage de l'enceinte villageoise à la muraille etc., tous ces aspects technologiques ont besoin d'être précisés. La multiplication des opérations d'archéologie du bâti et des fouilles sur les composantes des enceintes des petites et moyennes villes, largement négligées jusqu'à présent, est susceptible d'apporter de grandes avancées en la matière.

Les travaux de réseaux urbains ne font que très rarement I'objet d'une prescription de suivi archéologique. Or nous ne pouvons que le déplorer, dans la mesure où ce suivi permet de documenter l'intégralité du profil des enceintes, le plus souvent au niveau des tours-portes, soit I'un des points névralgiques des systèmes défensifs, de préciser la chronologie de leur fonctionnement et de leur abandon. Il constitue d'ailleurs l'une des rares occasions d'observer les vestiges des tours-portes détruites. Les suivis de réseaux réalisés par des archéologues bénévoles à Westhoffen, Mutzig et Neuwiller-lès-Saverne ont largement montré l'intérêt de cette pratique.

L'archéologie expérimentale pourrait également être sollicitée, pour appréhender des aspects comme la manipulation des armes sur le chemin de ronde.

\section{DE L'INTÉRÊT DES CORRÉLATIONS AVEC LES PETITES ET MOYENNES VILLES VOISINES}

L'étude réalisée en moyenne Alsace demanderait à être étendue aux enceintes urbaines d'un espace élargi au
Rhin supérieur. Il s'agirait de déterminer si les conclusions valables pour notre fenêtre, en termes chronologiques et typologiques, peuvent y être étendues. Mais aucun travail de synthèse sur ces enceintes des petites et moyennes villes en rive droite du Rhin n'a pour I'heure été entrepris. Nous livrons ici quelques pistes, issues de nos lectures monographiques, de l'observation de la documentation iconographique, de nos visites.

Ces localités d'outre-Rhin semblent avoir été dotées plus précocement d'une enceinte qu'en Alsace, la majorité se situant entre 1200 et 1300 , alors que la majorité pour I'Alsace oscille entre 1250 et 1350 . La nature exacte des enceintes précoces, avant tout citées par les textes, doit toutefois être précisée.

Mais de nombreux seigneurs alsaciens ayant été possessionnés dans le pays de Bade, ont pris part à son urbanisation et ont donc contribué à des transferts de pratiques d'une rive à I'autre. Les seigneurs de Lichtenberg ont par exemple fortifié Willstätt, tandis que les évêques de Strasbourg Conrad III et Jean I ${ }^{\text {er }}$ de Dirpheim ont respectivement entouré d'une muraille Lichtenau (vers 1293-1296) et amélioré les fortifications d'Oberkirch (entre 1306 et 1328).

De fait, il semble y avoir, au premier abord, une grande parenté entre les enceintes urbaines situées de part et d'autre du Rhin avec, par exemple, une belle convergence perceptible dans les dispositifs d'entrée, formés presque exclusivement de tours-portes.

De même, en particulier grâce aux gravures de Merian, on peut noter que de nombreuses localités ont fait l'objet de renforcements : la muraille de Gengenbach et celle de Villingen sont par exemple doublées par une braie formée d'un rempart revêtu. À Durlach et Zell-am-Hammersbach, une fausse-braie se déploie aussi au pied de la muraille.

Mais pour tous ces éléments, les données chronologiques restent à établir, ce qui rend impossible pour l'instant toute réflexion sur la nature et la vitesse des réponses apportées de part et d'autre du Rhin au développement de l'artillerie à poudre dans le cas de ces villes petites et moyennes.

\section{DE L'INTÉRÊT DES CORRÉLATIONS AVEC LES GRANDES VILLES VOISINES}

Ces questions relatives aux transferts des technologies se posent également entre grandes et petites villes. Une comparaison approfondie entre les enceintes de notre corpus et celles des villes voisines de taille respectable présenterait un grand intérêt, une démarche que nous n'avons toutefois pas pu mener dans le temps imparti à notre thèse. Il s'agit des anciens chefs lieux de cité (Strasbourg, Bâle) ou des villes d'origine médiévale à fort rayonnement économique ou politique (Colmar, Haguenau, Sélestat, Wissembourg, Fribourgen-Brisgau, etc.), qui tous ont fait peu ou prou l'objet de recherches développées sur leurs enceintes respectives. 
En matière de renforcement d'enceintes, Strasbourg apparaît précurseur en la matière, installant une deuxième muraille avec fossé devant son front nord, peut-être dès le $\mathrm{xIII}^{\mathrm{E}}$ siècle. C'est ce modèle qu'a pu suivre Obernai au début du XIVE siècle.

Mais pour ce qui est du recours à des braies remparées ou des systèmes de défense avancée de porte, et de manière plus générale, de l'adaptation à l'usage de l'artillerie en matière de défense tant passive qu'active, on ne peut pas vraiment faire état de différences chronologiques significatives entre ces grandes et petites villes. Il est vrai que nous manquons singulièrement d'études sérieuses, hors Strasbourg et Fribourg, sur la nature et le rythme de ces modernisations.

L'enceinte de Fribourg-en-Brisgau ne semble pas avoir été renforcée par l'adjonction d'une nouvelle ligne de défense (avant la construction d'un système bastionné au XVII ${ }^{\mathrm{E}}$ siècle). À Strasbourg, l'installation d'un rempart revêtu débute dans le dernier quart du XVe siècle. L'installation d'une barbacane (Vorwerk) y est aussi attestée dans le deuxième quart du $\mathrm{XV}^{\mathrm{e}}$ siècle, devant la porte Blanche du faubourg ouest, mais il faut attendre 1508-1510 pour que les deux autres portes du même faubourg soient renforcées par I'adjonction d'un ouvrage avancé (Bollwerk) [3].

Au total donc, la chronologie des adaptations aux armes à feu paraît concomitante entre grandes et petites villes, en tout cas sans différences majeures. Les structures mises en œuvre dans les grandes villes peuvent cependant se singulariser par leur ampleur : on note ainsi une largeur plus importante des fossés strasbourgeois, avec une moyenne de 25 à $30 \mathrm{~m}$.

\section{DE LA CAPACITÉ DE RÉSISTANCE DES ENCEINTES}

Nous avons tenté d'évaluer le potentiel défensif des enceintes des différentes villes du corpus, et ce pour deux dates différentes. En 1400, la très grande majorité des murailles de notre aire d'étude sont érigées et les enceintes n'ont généralement pas encore subi de transformations liées au développement de l'artillerie à poudre. À l'inverse, en 1590, elles ont toutes atteint peu ou prou leur développement maximal, à l'exception de Benfeld et Dachstein qui seront encore dotées d'une enceinte bastionnée dans les années qui suivirent.

Pour cette évaluation, nous avons établi un barème chiffré de divers éléments défensifs (hauteur muraille, profondeur fossé, distance entre les tours, défenses naturelles, etc.), dont la somme donne une note sur la qualité défensive [4].

Neuf critères ont été retenus pour 1400, jugés non seulement les plus pertinents quant à la capacité défensive intrinsèque de l'enceinte, mais pouvant aussi être renseignés pour le plus grand nombre de sites. À titre d'exemple, en ce qui concerne les fossés, seule leur largeur à l'ouverture a été retenue, les profondeurs étant mal documentées et les contrescarpes maçonnées présentant des problèmes de datation. Les enceintes de 28 villes dont nous avons pu établir un classement se situent ainsi avec une note (arrondie) entre 2 et 14, regroupées en 6 classes, de la plus faible (1, un cas, Boersch) aux plus fortes (5, Zellenberg, Guémar, et 6 , Molsheim, Obernai). L'essentiel des villes (17) se situe dans la fourchette des classes 2-3.

Nos résultats doivent évidemment être nuancés, les critères retenus pouvant être par trop empiriques. Mais une tendance se dégage toutefois, conforme à l'appréciation générale que peuvent avoir le visiteur et le chercheur aujourd'hui.

Le même exercice a été tenté pour 1590 , en ajoutant des critères tenant compte des évolutions en matière défensive (barbacane, rempart de terre, etc.). Nous avons exclu du choix les enceintes de Dachstein et de Benfeld, qui se voient dotées d'un premier système bastionné vers cette époque et qui relèvent d'une toute autre catégorie défensive. Le total nous permet maintenant d'observer 9 classes de villes. Les enceintes dont la «valeur défensive » estimée est de rang cinq à neuf sont assez logiquement presque toutes des enceintes complétées par un rempart (à l'exception des enceintes de Zellenberg, Molsheim et Mutzig). À l'inverse, des enceintes qui n'ont pas été renforcées par I'adjonction d'une nouvelle ligne de défense ou par un ouvrage de défense avancée de porte n'ont pas vu leur rang évoluer. Bien évidemment, des « reclassements » s'opèrent également entre les deux périodes : si Guémar et Obernai restent en tête, Niedernai se rapproche du haut du classement (classe 7) alors que d'autres reculent (Zellenberg qui reste au niveau 4 ).

Pour relativiser ces classements, il nous semble important de rappeler que le système défensif le plus complet, s'il est assurément dissuasif, n'est cependant que de peu d'utilité face à une troupe organisée et déterminée, s'il ne dispose pas d'effectifs suffisants, équipés et entraînés pour assurer son bon fonctionnement. Ce qui pose notamment la question du nombre d'hommes nécessaires pour veiller sur une enceinte et la défendre efficacement.

\section{[3] SCHWIEN 1992, p. 139.}

[4] Cette méthode a été extrapolée du travail de recherche effectué à l'université de Trèves sur le développement urbain dans un large espace centré sur Trèves (dont l'Alsace), chaque structure urbaine (enceinte, foire, marché, hôpital...) se voyant attribuer une note par période, dont le total permet de mesurer le degré d'« urbanité » par tranche de 50 ans entre 1000 et 1350. Voir : EsCher-HiRSCHMANn 2005. Pour notre tableau des qualités défensives des enceintes du corpus d'étude, voir VuiLLEMIN 2015, vol. 1, p. 340-341. 


\section{DU RÔLE POLITIQUE ET SOCIAL DE L'ENCEINTE}

L'enceinte constitue l'un des aspects majeurs de l'organisation sociale et politique de la ville. Car s'enfermer derrière une muraille n'est, en effet, pas sans conséquence pour les habitants. D'un point de vue très pragmatique, il faut avoir entrepris la visite de Boersch pour se rendre compte combien la communication entre l'intérieur et l'extérieur de ce qui était autrefois une ville médiévale est aujourd'hui encore malaisée, tant les points de passage sont peu nombreux, et en conséquence pour se faire une idée des contraintes auxquelles ont accepté de s'astreindre les habitants en décidant d'ériger une muraille. Le développement urbanistique est de fait lourdement conditionné par celle-ci.

Ces contraintes, si elles ont pu être la source de tensions par moment, ont sans nul doute contribué également au renforcement des liens de la communauté d'habitants. Ce corset de pierre peut participer sur le long terme à l'émancipation des bourgeois de la tutelle seigneuriale. Les fortifications font d'ailleurs partie des éléments symbolisant l'identité de la ville : elles peuvent être représentées sur les sceaux des villes, comme c'est le cas pour Masevaux et Bergheim.

L'enceinte urbaine participe au rayonnement de la ville sur ses environs et lui confère un pouvoir certain. En cas $d^{\prime}$ « éminents périls », la protection qu'offre la muraille attire les villageois. En Lorraine et en Franche-Comté, le droit des « retrahants», autorisant les villageois à se réfugier derrière les murailles des villes ou des châteaux, est pour ainsi dire règlementé au Xve siècle. En 1408, par exemple, le duc de Bourgogne Jean Sans Peur promulgue une ordonnance dans laquelle il fixe les devoirs réciproques des seigneurs détenteurs d'enceintes et des « retrahants » en Franche-Comté : les seigneurs ont obligation d'accueillir les « retrahants » qui en contrepartie doivent prendre part à la garde et aux travaux d'entretien [5]. Cette ordonnance restera en vigueur jusqu'à la fin de l'époque moderne. En Alsace, sans doute en raison du morcellement politique, la pratique du refuge n'est pas organisée à l'échelle régionale. Elle ne fait pourtant aucun doute.

\section{DE LA LONGÉVITÉ DES ENCEINTES URBAINES D'ORIGINE MÉDIÉVALE}

$\mathrm{Si}$, à l'exception de Dachstein et de Benfeld, les villes petites et moyennes n'ont pas pu évoluer vers le bastionnement, elles ont néanmoins continué à entretenir leurs structures défensives héritées du Moyen Âge. Lorsque Louis XIV ordonne en 1673 la réalisation de brèches dans les murailles des villes moyennes de la Décapole, c'est certes pour faire montre de son pouvoir, mais c'est peut-être aussi parce que ces villes constituent encore des menaces réelles à ses ambitions territoriales. Et de fait, il apparaît dans les mémoires rédigés vers 1779 que bon nombre d'entre elles peuvent encore être considérées comme des points d'appui dans la défense de la région par l'administration royale. Le déclassement d'Obernai comme place militaire, avec un gouverneur à sa tête, n'intervient qu'à l'extrême fin du XVIII ${ }^{\mathrm{e}}$ siècle (peu avant 1791).

Le déclin de la fonction défensive ne signifie d'ailleurs pas la perte de la fonction sécuritaire des enceintes, et ce jusque dans le courant du XIX ${ }^{e}$ siècle, et ouvre la voie à la réaffectation d'une partie de leurs composantes.

Le XIX ${ }^{\mathrm{e}}$ siècle s'avère nettement plus destructeur pour les enceintes urbaines que les deux siècles précédents. Mû tant par les thèses hygiénistes, les projets urbanistiques, les plans de rationalisation du tracé des rues et des routes, que par la volonté pour les municipalités de se décharger de l'entretien coûteux de ces structures, le démantèlement des fortifications est entrepris.

Les démantèlements ne sont pas sans susciter des oppositions internes aux villes. Il serait intéressant d'approfondir la question des forces contraires - destruction et conservation - qui tiraillent les choix à faire pour les enceintes au $\mathrm{XIX}^{\mathrm{e}}$ siècle et $\mathrm{au} \mathrm{XX}^{\mathrm{e}}$ siècle. Une telle étude, en plus d'illustrer la mise en place des institutions en charge de la sauvegarde du patrimoine en France (et en Allemagne) et le développement des sociétés érudites, relève également de l'histoire politique, sociale et des mentalités.

[5] BOUVARD 2007, p. 16-17. 


\section{BIBLIOGRAPHIE}

Bouvard, André, 2007, «Les retrahants aux $\mathrm{XV}^{\mathrm{e}}$ et $\mathrm{xVI} \mathrm{e}^{\mathrm{e}}$ siècles », in coll., Hommes d'armes et gens de guerre, du Moyen Âge au $x V I I^{e}$ siècle, Franche-Comté de Bourgogne et comté de Montbéliard, Besançon, p. 16-17.

Escher-Apsner, Monika \& Hirschmann, Frank G. (éd.), 2005, Die urbanen Zentren des hohen und späteren Mittelalters: vergleichende Untersuchungen zu Städten und Städtelandschaften im Westen des Reiches und in Ostfrankreich, Trier, 3 vol.

Henigfeld, Yves \& Masquilier, Amaury (dir.), 2008, Archéologie des enceintes urbaines et leurs abords en Lorraine et en Alsace ( $X I I^{e}-X V^{e}$ siècle), Dijon (Revue Archéologique de I'Est, 26e suppl.).

Metz, Bernhard, 1991-2005, «Alsatia munita aevii medii. Répertoire critique des sites fortifiés de l'ancienne Alsace du $10^{\mathrm{e}}$ siècle à la Guerre de Trente Ans », Société pour la Conservation des Monuments Historiques d'Alsace. Bulletin d'information, n 1 à 34 [6]. Metz, Bernhard, 2002, «Essai sur la hiérarchie des villes médiévales d'Alsace (1200-1350) - 1 ère partie », Revue $d^{\prime}$ Alsace $n^{\circ} 128$, p. 47-100.

Metz, Bernhard, 2008, «Essai sur la hiérarchie des villes médiévales d'Alsace (1200-1350) - 2e partie », Revue d'Alsace $n^{\circ} 134$, p. $129-167$.

Schwien, Jean-Jacques, 1992, Strasbourg : document d'évaluation du patrimoine archéologique urbain, Paris.

Vuillemin, Adrien, 2009, Les fortifications villageoises entre la Zorn et la Bruche (XII ${ }^{e}-X V I^{e}$ siècles) : un phénomène historique au regard des statistiques et du parcellaire, Mémoire de master sous la direction de G. Bischoff, Université de Strasbourg, 2 vol.

Vuillemin, Adrien, 2013, «L'enceinte urbaine d'Ingwiller (Bas-Rhin) de 1345 à 2013 », Cahiers Alsaciens d'Archéologie, d'Art et d'Histoire 58, p. 123-144.

Vuillemin, Adrien, 2015, Enceintes urbaines en moyenne Alsace. Thèse d'histoire et d'archéologie médiévale, sous la direction de G. Bischoff et J.-J. Schwien, Université de Strasbourg, 3 vol.

[6] Ensemble de notices par livraison semestrielle. Ce projet est aujourd'hui inabouti. Il est accessible en ligne sur le site web http://www.scmha.fr/ressources/alsatia-munita. 